\title{
Efeito da aplicação de diferentes revestimentos comestíveis na conservação de maçãs 'Royal Gala' minimamente processadas
}

\section{Effect of applying different edible coatings on conservation of minimally processed 'Royal Gala' apples}

\author{
Sandriane Pizato ${ }^{1 *}$; William Renzo Cortez-Vega ${ }^{2}$; \\ Carlos Prentice-Hernández ${ }^{3}$; Caroline Dellinghausen Borges ${ }^{4}$
}

\begin{abstract}
Resumo
O objetivo deste estudo foi avaliar os efeitos da aplicação de diferentes gomas (tara, xantana e alginato de sódio) associado com ácido cítrico, ascórbico e cloreto de cálcio, em relação as características químicas, físicas e microbiológicas na conservação de maçãs 'Royal Gala' minimamente processadas quando armazenadas a $4 \pm 1{ }^{\circ} \mathrm{C}$. As maçãs foram lavadas, descascadas, cortadas em cubos e armazenadas em embalagem PET, por um período de 15 dias a $4 \pm 1^{\circ} \mathrm{C}$. Realizaram-se análises de perda de massa, firmeza, cor $\left(\mathrm{L}^{*} \mathrm{e} \mathrm{h}^{*}\right), \mathrm{pH}$, sólidos solúveis totais, e análises microbiológicas (psicrófilos, bolores e leveduras, Salmonella e Escherichia coli). As análises foram realizadas em triplicata no dia do processamento das amostras (dia 0) e após 1, 3, 5, 7, 9, 12 e 15 dias de armazenamento. Pode-se concluir que os diferentes revestimentos utilizados neste trabalho foram eficientes na conservação de maçãs minimamente processadas, quando comparado a amostra controle. Dos revestimentos avaliados, o tratamento com goma xantana, associado aos ácidos cítrico, ascórbico e do cloreto de cálcio foi o que apresentou os melhores resultados, mostrando-se capaz de reduzir a perda de massa, o crescimento microbiano, manter a cor e a firmeza das maçãs minimamente processadas.
\end{abstract}

Palavras-chave: Tara, xantana, alginato, análises físico-químicas, análises microbiológicas

\begin{abstract}
The objective of this study was to evaluate the effects of applying different gums (tara, xanthan and sodium alginate) associated with citric acid, ascorbic acid and calcium chloride, in relation to chemical, physical and microbiological characteristics in the conservation of minimally processed Royal Gala apples when stored at $4 \pm 1{ }^{\circ} \mathrm{C}$. The apples were washed, peeled, diced and stored in a PET packaging for a period of 15 days at $4 \pm 1{ }^{\circ} \mathrm{C}$. Analyses of weight loss, firmness, color ( $\left.\mathrm{L}^{*} \mathrm{e} \mathrm{h}\right)$, pH, total soluble solids, and microbiological analyses (psychrophyls, yeasts and molds, Salmonella and Escherichia coli) were carried out. The analyses were performed in triplicate on the processing day (day 0 ) of the samples and after 1, 3, 5, 7, 9, 12 and 15 days of storage. It can be concluded that the different coatings used in this study were effective in preserving minimally processed apples, as compared to the control sample. Among the evaluated coatings, treatment with xanthan gum, associated with citric acid, ascorbic acid and calcium chloride showed the best results, being capable of reducing the weight loss, microbial growth, keeping the firmness and color of minimally processed apples.
\end{abstract}

Key words: Tara, xanthan, alginate, physicochemical analyses, microbiological analyses

1 Discente de Pós-Graduação em Engenharia e Ciência de Alimentos, Universidade Federal do Rio Grande, FURG, Rio Grande, RS. E-mail: sandrianepizato@yahoo.com.br

2 Prof. Adjunto, Universidade Federal da Grande Dourados, UFGD, Dourados, MS. E-mail: williamvega@ufgd.edu.br

3 Prof. Associado, FURG, Rio Grande, RS. E-mail: dqmprent@furg.br

4 Prof ${ }^{a}$ Adjunta, FURG, Rio Grande, RS. E-mail: caroldellin@bol.com.br

Autor para correspondência 


\section{Introdução}

A demanda por frutas e hortaliças minimamente processadas vem crescendo no mercado alimentício, tendo em vista o desejo do consumidor por alimentos que mantenham seu frescor e características próximas ao in natura (JUNQUEIRA et al., 2009). No entanto, produtos minimamente processados têm uma vida-útil relativamente curta, devido a cortes e à sua manipulação, o que ocasiona aumento do seu metabolismo (PERERA et al., 2010).

O processamento mínimo da maçã representa uma forma de aproveitar excedentes de produção, agregar valor ao produto e ainda propiciar a conveniência do consumo (VILAS-BOAS et al., 2009). Entretanto, a principal dificuldade observada neste processo, é minimizar os efeitos da atividade enzimática, em decorrência da exposição ao ar, que ocasiona o escurecimento do produto. Além desta alteração, outras são passíveis de ocorrência como perda de umidade, textura e crescimento microbiano (FONTES et al., 2008). Uma alternativa para reduzir os efeitos causados pelo processo mínimo é o uso de revestimentos comestíveis (KROCHTA; MULDERJOHNSTON, 1997). Polissacarídeos, proteínas e/ ou lipídeos têm sido utilizados como revestimentos comestíveis, a estes revestimentos podem ser adicionados agentes antioxidantes, antimicrobianos, entre outros. Neste caso o revestimento promove a imobilização do composto ativo no polímero, o que pode manter a alta concentração do composto na superfície do alimento (OUATTARA; SABATO; LACROIX, 2001).

A goma tara é extraída do endosperma da semente da Caesalpinia spinosa e utilizada em alimentos como agente espessante (SITTIKIJYOTHIN; TORRES; GONÇALVES, 2005). Esta goma é nativa da região dos Andes da Bolívia, Peru e norte do Chile, sendo o Peru, o maior exportador. Apresenta um custo relativamente baixo, proporcionando um grande incentivo econômico para sua exploração industrial (FOOD STANDARDS AUSTRALIA NEW ZEALAND - FSANZ, 2006), sendo que ainda não foi avaliada como revestimento comestível de alimentos minimamente processados.

A goma xantana é um polissacarídeo sintetizado por uma bactéria fitopatogênica do gênero Xanthomonas. Esta goma é utilizada em alimentos como agente espessante e estabilizante, entretanto isoladamente esta goma não forma gel, apenas em sinergismo com galactomananas (GARCÍAOCHOA et al., 2000).

$\mathrm{O}$ alginato de sódio é um polissacarídeo de origem natural derivado, principalmente, de algas marinhas marrons. A presença de cátions polivalentes fazem com que ocorra a gelificação do alginato, sendo os sais de cálcio, os agentes gelificantes mais efetivos (MENEGUEL; BENASSI; YAMASHITA, 2008).

Assim, objetivo deste estudo foi avaliar os efeitos da aplicação de diferentes revestimentos (gomas tara, xantana ou alginato de sódio) associado a agente de firmeza e antioxidantes na conservação de maçãs 'Royal Gala' minimamente processadas, armazenadas a $4 \pm 1{ }^{\circ} \mathrm{C}$.

\section{Material e Métodos}

\section{Material}

Foram utilizadas maçãs da cultivar 'Royal Gala'. As frutas foram selecionadas, considerando a ausência de defeitos fisiológicos, tamanho e cor. Estas foram transportadas em caixas térmicas até o Laboratório de Tecnologia de Alimentos da Universidade Federal do Rio Grande, onde foi realizado o processamento mínimo.

\section{Preparo das maçãs minimamente processadas}

$\mathrm{O}$ processamento foi realizado à temperatura de, aproximadamente, $10{ }^{\circ} \mathrm{C}$, com os utensílios previamente higienizados em solução de cloro orgânico (dicloroisocianurato de sódio), na concentração de 2 g.L. ${ }^{-1}$. As maçãs selecionadas foram lavadas e sanitizadas em solução de cloro orgânico na concentração de 2 g.L. L $^{-1}$ por 15 minutos, 
descascadas e cortadas manualmente em pedaços de, aproximadamente, $2,5 \times 2,5 \mathrm{~cm}$, com auxílio de facas de aço inoxidável. Em seguida os pedaços foram enxaguados com água clorada $\left(0,2\right.$ g. $\left.\mathrm{L}^{-1}\right)$ para eliminar o suco celular extravasado.

\section{Preparo e aplicações dos revestimentos}

As gomas xantana (Shandong Fufeng), tara (Silva Extract Srl) e o alginato de sódio (Kimica) foram dissolvidos, lentamente, em água à temperatura de $25^{\circ} \mathrm{C}$, sob agitação constante por, aproximadamente, 1 hora. A goma xantana seguiu ao aquecimento a $60{ }^{\circ} \mathrm{C}$ por 20 minutos (XUEWU et al., 1996), a goma tara a $80{ }^{\circ} \mathrm{C}$ por 30 minutos (SITTIKIJYOTHIN; SAMPAIO; GONÇALVES, 2007) e o alginato de sódio a $70{ }^{\circ} \mathrm{C}$ por 30 minutos, sendo após resfriado até $15{ }^{\circ} \mathrm{C}$ (ROJAS-GRAÜ et al., 2007). Após o resfriamento das soluções foi adicionado sob agitação o cloreto de cálcio, os ácidos cítrico e ascórbico e o plastificante glicerol. Os revestimentos utilizados foram preparados em solução aquosa: Tratamento 1 - Controle (maçã cortada); Tratamento 2 - alginato $(2 \% \mathrm{p} / \mathrm{v})$, ácido ascórbico $(1 \% \mathrm{p} / \mathrm{v})$, ácido cítrico $(0,25 \% \mathrm{p} / \mathrm{v})$, $\mathrm{CaCl}_{2}(1 \% \mathrm{p} / \mathrm{v})$ e glicerol $(1 \% \mathrm{v} / \mathrm{v})$; Tratamento 3 - goma xantana $(0,5 \% \mathrm{p} / \mathrm{v})$, ácido ascórbico ( $1 \%$ $\mathrm{p} / \mathrm{v})$, ácido cítrico $(0,25 \% \mathrm{p} / \mathrm{v}), \mathrm{CaCl}_{2}(1 \% \mathrm{p} / \mathrm{v})$ e glicerol $(1 \% \mathrm{v} / \mathrm{v})$; Tratamento 4 - goma tara $(0,5 \%$ $\mathrm{p} / \mathrm{v})$, ácido ascórbico (1\% p/v), ácido cítrico $(0,25$ $\% \mathrm{p} / \mathrm{v}), \mathrm{CaCl}_{2}(1 \% \mathrm{p} / \mathrm{v})$ e glicerol $(1 \% \mathrm{v} / \mathrm{v})$.

Os pedaços foram totalmente submersos nas soluções por 1 minuto e, em seguida drenados utilizando telas de nylon, por 2 a 3 minutos para que o excesso de solução fosse eliminado. Os pedaços foram secos com auxílio de ventilador, em ambiente refrigerado, por 30 minutos. Por fim, as amostras foram embaladas em embalagem não reciclada de PET - Polietileno Tereftalato, com tampa (SANPACK), cujas medidas externas são de $15,5 \times 13,2 \times 5,5 \mathrm{~cm}$, padronizando o número de pedaços por embalagem ( 8 pedaços) e armazenadas em condições refrigeradas, a $4 \pm 1{ }^{\circ} \mathrm{C}$ durante 15 dias. As análises físicas, físico-químicas e microbiológicas foram realizadas em triplicata no dia do processamento das amostras (dia 0) e após 1 , $3,5,7,9,12$ e 15 dias de armazenamento.

\section{Análises físicas e físico-químicas}

\section{Perda de massa}

A perda de massa foi obtida considerando-se a diferença entre o peso inicial da maçã minimamente processada e aquele obtido ao final de cada tempo de armazenamento, de acordo com a fórmula: Perda de massa $=[($ massa inicial - massa final $) /($ massa inicial)] x 100. Os resultados foram expressos em porcentagem de perda de massa.

\section{Análise de firmeza (N)}

As medidas de firmeza dos pedaços de maçã foram determinadas utilizando-se um texturômetro (Stable Micro Systems modelo TA.XTplus). Foi utilizado a ponteira cilíndrica $(25 \mathrm{~mm} \mathrm{P} / 25 \mathrm{P}) \mathrm{em}$ velocidade de pré-teste de $4 \mathrm{~mm} \cdot \mathrm{s}^{-1}$, pós-teste de $8 \mathrm{~mm} . \mathrm{s}^{-1}$, teste de $2 \mathrm{~mm} . \mathrm{s}^{-1}$; e profundidade de penetração de $5 \mathrm{~mm}$. Os resultados foram expressos em Newton $(\mathrm{N})$.

\section{Cor}

As análises de cor foram realizadas utilizandose um colorímetro (Minolta, modelo Chroma Meter CR400). Foram medidos os parâmetros de luminosidade $\mathrm{L}^{*}$ [0 (preto) a 100 (branco)], a* [cromaticidade do verde (-60) a vermelho $(+60)$ ], b* [cromaticidade do azul (-60) para amarelo $(+60)$ ]. Os valores de $\mathrm{a}^{*} \mathrm{e} \mathrm{b}^{*}$ foram utilizados para calcular a tonalidade cromática $(H u e)\left[{ }^{\circ} \mathrm{h}^{*}=\arctan \left(\mathrm{b}^{*} / \mathrm{a}^{*}\right)\right]$, o qual mostra a localização da cor em um diagrama, onde o ângulo $0^{\circ}$ representa vermelho puro, $90^{\circ}$ representa o amarelo puro, $180^{\circ}$ o verde puro e $270^{\circ}$ o azul (CARDOSO et al., 2007). 


\section{Análise de $\mathrm{pH}$}

Para medida do $\mathrm{pH}, 20 \mathrm{~g}$ das amostras foram trituradas em $100 \mathrm{~mL}$ de água destilada, em seguida realizou-se a medição utilizando um pHmetro (Marconi PA 200). A análise foi realizada segundo o método descrito pela AOAC (2000).

\section{Teor de sólidos solúveis totais (Brix)}

Os teores de sólidos solúveis totais foram determinados a partir do extrato líquido obtido após a trituração da amostra. Utilizou-se um refratômetro de bancada (marca Abbe, UK), sem controle automático de temperatura. Os resultados foram expressos em Brix (AOAC, 2000).

\section{Análises microbiológicas}

Para as análises microbiológicas, adicionou-se $225 \mathrm{~mL}$ de água peptonada $0,1 \%(\mathrm{p} / \mathrm{v})$ estéril a 25 $\mathrm{g}$ de amostra. Em seguida foram homogeneizadas em Blenders por 60 segundos e feitas sucessivas diluições em água peptonada $0,1 \%(\mathrm{p} / \mathrm{v})$ estéril.
As diluições das amostras foram selecionadas, baseando-se na contagem de micro-organismos esperada para cada amostra.

Os testes microbiológicos realizados foram psicrófilos spp., Escherichia coli, bolores e leveduras e Salmonella spp., seguindo a metodologia descrita na APHA (2001) e Brasil (2001).

\section{Avaliação estatística}

Os resultados obtidos das avaliações físicas e físico-químicas foram submetidos à análise de variância e a comparação de médias foi realizada pelo Teste de Tukey com nível de significância de $5 \%$, utilizando-se o programa Statistica 7.0.

\section{Resultados e Discussões}

A Tabela 1 apresenta os valores de perda de massa para maçãs minimamente processadas utilizando diferentes revestimentos e armazenadas a $4 \pm 1{ }^{\circ} \mathrm{C}$ por 15 dias.

Tabela 1. Perda de massa (\%) de maçãs minimamente processadas tratadas com diferentes revestimentos e armazenadas a $4 \pm 1^{\circ} \mathrm{C}$ durante 15 dias.

\begin{tabular}{ccccc}
\hline \multirow{2}{*}{ DIAS } & \multicolumn{4}{c}{ TRATAMENTO } \\
\cline { 2 - 5 } & T1 & T2 & T3 & T4 \\
\hline 0 & $0^{\mathrm{hA}}$ & $0^{\mathrm{hA}}$ & $0^{\mathrm{hA}}$ & $0^{\mathrm{hA}}$ \\
1 & $0,68 \pm 0,07^{\mathrm{gB}}$ & $0,48 \pm 0,02^{\mathrm{gC}}$ & $0,63 \pm 0,02^{\mathrm{gB}}$ & $1,01 \pm 0,06^{\mathrm{gA}}$ \\
3 & $2,16 \pm 0,05^{\mathrm{fA}}$ & $1,43 \pm 0,07^{\mathrm{fB}}$ & $1,34 \pm 0,03^{\mathrm{fB}}$ & $2,11 \pm 0,02^{\mathrm{fA}}$ \\
5 & $3,24 \pm 0,03^{\mathrm{eA}}$ & $1,97 \pm 0,03^{\mathrm{eD}}$ & $2,29 \pm 0,02^{\mathrm{eC}}$ & $2,91 \pm 0,05^{\mathrm{eB}}$ \\
7 & $4,32 \pm 0,02^{\mathrm{dA}}$ & $2,68 \pm 0,02^{\mathrm{dD}}$ & $3,09 \pm 0,01^{\mathrm{dC}}$ & $3,92 \pm 0,03^{\mathrm{dB}}$ \\
9 & $5,14 \pm 0,04^{\mathrm{cB}}$ & $3,40 \pm 0,04^{\mathrm{cD}}$ & $3,96 \pm 0,08^{\mathrm{cC}}$ & $5,32 \pm 0,02^{\mathrm{cA}}$ \\
12 & $6,49 \pm 0,02^{\mathrm{bA}}$ & $4,59 \pm 0,02^{\mathrm{bD}}$ & $5,22 \pm 0,02^{\mathrm{bC}}$ & $6,42 \pm 0,02^{\mathrm{bB}}$ \\
15 & $8,78 \pm 0,02^{\mathrm{aA}}$ & $5,84 \pm 0,01^{\mathrm{aD}}$ & $7,28 \pm 0,07^{\mathrm{aC}}$ & $7,53 \pm 0,01^{\mathrm{aB}}$ \\
\hline
\end{tabular}

Médias seguidas de mesma letra minúscula na coluna e maiúscula na linha não diferem entre si, pelo Teste de Tukey (P<0,05). (T1) controle; (T2) alginato $2 \%$, ácido ascórbico $1 \%$, ácido cítrico 0,25\%, $\mathrm{CaCl}_{2} 1 \%$, glicerol $1 \%$; (T3) goma xantana $0,5 \%$, ácido ascórbico $1 \%$, ácido cítrico $0,25 \%, \mathrm{CaCl}_{2} 1 \%$, glicerol $1 \%$; (T4) goma tara $0,5 \%$, ácido ascórbico $1 \%$, ácido cítrico 0,25 $\%, \mathrm{CaCl}_{2} 1 \%$, glicerol $1 \%$.

Fonte: Elaboração dos autores. 
Houve aumento da perda de massa durante o período de armazenamento em todos os tratamentos estudados. A perda de massa obtida nas amostras do tratamento controle $\mathrm{T} 1$, foi significativamente superior aos demais tratamentos, chegando a $8,78 \%$ em 15 dias de armazenamento. Dentre as amostras revestidas, aquelas submetidas ao tratamento $\mathrm{T} 2$, com alginato de sódio, obtiveram a menor perda de massa (5,8\%). A perda de massa ocorre, principalmente, pela transpiração e também pelo tempo de armazenamento (CARVALHO; LIMA, 2002). Os alginatos são os biopolímeros mais utilizados como revestimentos comestíveis, pois são agentes gelificantes que formam géis fortes devido a reação com cátions como o cálcio. Neste estudo o agente gelificante alginato de sódio foi mais eficiente em manter a umidade no produto do que os agentes espessantes goma xantana e tara.

Os resultados obtidos no presente trabalho foram inferiores aos encontrados por Olivas, Mattinson e Barbosa-Cánovas (2007), ao avaliar a perda de massa de maçãs minimamente processadas revestidas com alginato de sódio, em 10 dias de armazenamento. Os melhores resultados foram obtidos com o revestimento composto de alginato, cloreto de cálcio e monoglicerídeo acetilado, obtendo-se $17,8 \%$ de perda de massa. Altos valores também foram obtidos no estudo de Qi et al. (2011) em que maçãs minimamente processadas revestidas com quitosana e armazenadas por 8 dias, perderam em média $15 \%$ de massa.

A Tabela 2 apresenta os resultados de firmeza encontrados para maçãs minimamente processadas utilizando diferentes revestimentos comestíveis, armazenadas à $4 \pm 1{ }^{\circ} \mathrm{C}$, por um período de 15 dias.

Houve aumento significativo da firmeza durante o armazenamento, nas amostras submetidas aos tratamentos T1 (26,8\%) e T2 (19,8\%). Segundo Souza et al. (2005), o aumento da firmeza deve-se à formação de um tecido superficial resistente, devido à maior perda de umidade, fazendo com que os pedaços tornem-se mais firmes (ressecamento dos pedaços) durante o armazenamento. Já nas amostras submetidas ao tratamento T4, a firmeza decaiu significativamente após o $5^{\circ}$ dia de armazenamento, alcançando redução de $10,7 \%$. Este comportamento é decorrente da atuação de enzimas, principalmente, enzimas pécticas como poligalacturonase (FONTES, 2005). Somente o revestimento composto por goma xantana (T3) manteve a firmeza inicial das maçãs minimamente processadas.

Tabela 2. Firmeza $(\mathrm{N})$ de maçãs minimamente processadas tratadas com diferentes revestimentos e armazenadas a $4 \pm 1^{\circ} \mathrm{C}$ durante 15 dias.

\begin{tabular}{|c|c|c|c|c|}
\hline \multirow{2}{*}{ DIAS } & \multicolumn{4}{|c|}{ TRATAMENTO } \\
\hline & T1 & T2 & T3 & T4 \\
\hline 0 & $32,64 \pm 1,32^{\mathrm{cB}}$ & $32,62 \pm 0,87^{\mathrm{cB}}$ & $42,19 \pm 1,78^{\mathrm{aA}}$ & $43,38 \pm 1,12^{\mathrm{aA}}$ \\
\hline 1 & $32,73 \pm 1,54^{\mathrm{cB}}$ & $32,65 \pm 1,16^{\mathrm{cB}}$ & $42,28 \pm 0,75^{\mathrm{aA}}$ & $43,38 \pm 0,23^{\mathrm{aA}}$ \\
\hline 3 & $35,56 \pm 1,89^{\mathrm{cB}}$ & $33,68 \pm 0,49^{\mathrm{cB}}$ & $42,38 \pm 1,23^{\mathrm{aA}}$ & $43,85 \pm 2,06^{\mathrm{aA}}$ \\
\hline 5 & $39,37 \pm 1,15^{\mathrm{bA}}$ & $35,72 \pm 0,27^{\mathrm{bcB}}$ & $42,69 \pm 2,54^{\mathrm{aA}}$ & $42,17 \pm 0,86^{\mathrm{abA}}$ \\
\hline 7 & $41,65 \pm 0,88^{\mathrm{abAB}}$ & $37,84 \pm 1,54^{\mathrm{abC}}$ & $42,81 \pm 0,72^{\mathrm{aA}}$ & $39,06 \pm 1,41^{\mathrm{bBC}}$ \\
\hline 9 & $41,98 \pm 0,92^{\mathrm{abAB}}$ & $38,21 \pm 1,31^{\mathrm{abC}}$ & $43,34 \pm 0,93^{\mathrm{aA}}$ & $38,92 \pm 1,67^{\mathrm{bBC}}$ \\
\hline 12 & $42,39 \pm 1,34^{\mathrm{abA}}$ & $38,56 \pm 1,25^{\mathrm{abB}}$ & $43,94 \pm 0,37^{\mathrm{aA}}$ & $38,81 \pm 0,62^{\mathrm{bB}}$ \\
\hline 15 & $44,59 \pm 0,77^{\mathrm{aA}}$ & $40,76 \pm 1,93^{\mathrm{aBC}}$ & $44,34 \pm 0,83^{\mathrm{aAB}}$ & $38,72 \pm 1,72^{\mathrm{bC}}$ \\
\hline
\end{tabular}

Médias seguidas de mesma letra minúscula na coluna e maiúscula na linha não diferem entre si, pelo Teste de Tukey (P<0,05). (T1) controle; (T2) alginato $2 \%(\mathrm{~m} / \mathrm{v})$, ácido ascórbico $1 \%(\mathrm{~m} / \mathrm{v})$, ácido cítrico $0,25 \%(\mathrm{~m} / \mathrm{v}), \mathrm{CaCl}_{2} 1 \%(\mathrm{~m} / \mathrm{v})$, glicerol $1 \%$ (v/v); (T3) goma xantana $0,5 \%(\mathrm{~m} / \mathrm{v})$, ácido ascórbico $1 \%(\mathrm{~m} / \mathrm{v})$, ácido cítrico $0,25 \%(\mathrm{~m} / \mathrm{v}), \mathrm{CaCl}_{2} 1 \%$ (m/v), glicerol $1 \%(\mathrm{v} / \mathrm{v}) ;(\mathrm{T} 4)$ goma tara $0,5 \%(\mathrm{~m} / \mathrm{v})$, ácido ascórbico $1 \%(\mathrm{~m} / \mathrm{v})$, ácido cítrico $0,25 \%(\mathrm{~m} / \mathrm{v}), \mathrm{CaCl}_{2} 1 \%(\mathrm{~m} / \mathrm{v})$, glicerol $1 \%(\mathrm{v} / \mathrm{v})$.

Fonte: Elaboração dos autores. 
Fontes et al. (2008) trabalhando com maçãs minimamente processadas revestidas com alginato de sódio e/ou fécula de mandioca, observaram endurecimento do tecido. Os autores atribuíram esse fato à adição de sal de cálcio na formulação do revestimento, pois os íons de cálcio formam complexos com a pectina da parede celular, melhorando a integridade estrutural e proporcionando maior firmeza dos tecidos. Qi et al. (2011) também verificaram que o revestimento de quitosana e tratamento com cloreto de cácio retardou o amolecimento do tecido em fatias de maçã.

Rojas-Graü, Tapia e Martín-Belloso (2008) avaliaram a firmeza de amostras de maçã minimamente processadas revestidas com alginato e gelana. De acordo com seus resultados, o cloreto de cálcio promoveu as ligações entre as cadeias dos polímeros, reduzindo desta forma a perda de firmeza. O efeito do cloreto de cálcio neste experimento não foi relevante, visto que houve aumento da firmeza na amostra controle que não foi tratada com cloreto de cálcio, sendo atribuída para isso uma maior perda de umidade.

A Tabela 3 mostra os valores de luminosidade (L*) em maçãs minimamente processadas com uso de diferentes revestimentos, armazenadas a $4 \pm 1{ }^{\circ} \mathrm{C}$ por 15 dias.

Em todos os tratamentos pode-se verificar um aumento do escurecimento ( $\left.\mathrm{L}^{*}\right)$, sendo que as amostras do tratamento controle $(9,0 \%)$ e aquelas submetidas ao tratamento T2 $(6,5 \%)$ apresentaram aumento significativo, quando comparado o primeiro e último dia de armazenamento. Diferente do comportamento observado nos tratamentos T3 e T4, em que o aumento não foi significativo, alcançando, $1,3 \%$ e $1,4 \%$, respectivamente. O cloreto de cálcio, em alguns casos, tem sido utilizado no controle do escurecimento, pois inibe a enzima polifenoloxidase pela interação do íon cloreto com o cobre do centro ativo desta enzima (GARCIA; BARRET, 2002). Além disto, o revestimento age como barreira ao oxigênio, necessário para as reações de escurecimento (FREITAS, 2010), fato este que pode ser observado no presente trabalho que, dependendo do revestimento, ocorreu menor redução da luminosidade.

Os resultados de luminosidade no presente trabalho foram semelhantes ao encontrados por Perez Gago, Serra e Del-Río (2006) que também demonstraram que o uso de antioxidantes proporcionou maior inibição do escurecimento enzimático.

Tabela 3. Luminosidade ( $\mathrm{L}^{*}$ ) de maçãs minimamente processadas tratadas com diferentes revestimentos e armazenadas a $4 \pm 1^{\circ} \mathrm{C}$ durante 15 dias.

\begin{tabular}{ccccc}
\hline \multirow{2}{*}{ DIAS } & \multicolumn{5}{c}{ TRATAMENTO } \\
\cline { 2 - 5 } & \multicolumn{1}{c}{ T1 } & \multicolumn{1}{c}{ T2 } & T3 & T4 \\
\hline 0 & $78,20 \pm 1,59^{\mathrm{aA}}$ & $80,5 \pm 0,84^{\mathrm{aA}}$ & $80,51 \pm 1,17^{\mathrm{aA}}$ & $80,50 \pm 0,72^{\mathrm{abA}}$ \\
1 & $76,57 \pm 1,25^{\mathrm{abB}}$ & $79,04 \pm 0,98^{\mathrm{abA}}$ & $80,30 \pm 1,14^{\mathrm{aA}}$ & $80,50 \pm 0,51^{\mathrm{abA}}$ \\
3 & $75,75 \pm 0,75^{\mathrm{abAB}}$ & $78,20 \pm 0,58^{\mathrm{bcAB}}$ & $80,30 \pm 0,71^{\mathrm{bB}}$ & $81,08 \pm 0,78^{\mathrm{aA}}$ \\
5 & $75,32 \pm 0,37^{\mathrm{bcD}}$ & $76,86 \pm 0,52^{\mathrm{cdC}}$ & $79,43 \pm 0,32^{\mathrm{aB}}$ & $80,92 \pm 0,47^{\mathrm{abA}}$ \\
7 & $74,45 \pm 0,71^{\mathrm{bcC}}$ & $76,65 \pm 0,35^{\mathrm{cdB}}$ & $79,45 \pm 0,61^{\mathrm{aA}}$ & $80,82 \pm 0,51^{\mathrm{abA}}$ \\
9 & $74,44 \pm 0,26^{\mathrm{bcC}}$ & $76,01 \pm 0,74^{\mathrm{dB}}$ & $79,46 \pm 0,53^{\mathrm{aA}}$ & $80,69 \pm 0,48^{\mathrm{abA}}$ \\
12 & $72,95 \pm 1,01^{\mathrm{cdC}}$ & $75,46 \pm 0,58^{\mathrm{dB}}$ & $79,45 \pm 0,29^{\mathrm{aA}}$ & $80,14 \pm 0,55^{\mathrm{abA}}$ \\
15 & $71,16 \pm 0,16^{\mathrm{dC}}$ & $75,23 \pm 0,53^{\mathrm{dB}}$ & $79,68 \pm 0,31^{\mathrm{aA}}$ & $79,38 \pm 0,14^{\mathrm{bA}}$ \\
\hline
\end{tabular}

Médias seguidas de mesma letra minúscula na coluna e maiúscula na linha não diferem entre si, pelo Teste de Tukey (P<0,05). (T1) controle; (T2) alginato $2 \%(\mathrm{~m} / \mathrm{v})$, ácido ascórbico $1 \%(\mathrm{~m} / \mathrm{v})$, ácido cítrico $0,25 \%(\mathrm{~m} / \mathrm{v}), \mathrm{CaCl}_{2} 1 \%(\mathrm{~m} / \mathrm{v})$, glicerol $1 \%$ (v/v); (T3) goma xantana $0,5 \%(\mathrm{~m} / \mathrm{v})$, ácido ascórbico $1 \%(\mathrm{~m} / \mathrm{v})$, ácido cítrico $0,25 \%(\mathrm{~m} / \mathrm{v}), \mathrm{CaCl}_{2} 1 \%(\mathrm{~m} / \mathrm{v})$, glicerol $1 \%$ (v/v); (T4) goma tara $0,5 \%(\mathrm{~m} / \mathrm{v})$, ácido ascórbico $1 \%(\mathrm{~m} / \mathrm{v})$, ácido cítrico 0,25\% (m/v), $\mathrm{CaCl}_{2} 1 \%(\mathrm{~m} / \mathrm{v})$, glicerol $1 \%(\mathrm{v} / \mathrm{v})$.

Fonte: Elaboração dos autores. 
$\mathrm{Na}$ Tabela 4 são apresentados os valores de tonalidade cromática $\left(\mathrm{h}^{*}\right)$ encontrados para maçãs minimamente processadas revestidas com diferentes coberturas armazenadas a $4 \pm 1{ }^{\circ} \mathrm{C}$ por 15 dias.

Todos os tratamentos apresentaram redução significativa do ângulo ao longo do armazenamento. A amostra controle foi a que obteve maior redução $(85,33 \%)$ de $h^{*}$, sendo os valores obtidos significativamente inferiores aos demais tratamentos. Já o tratamento T3 foi o que obteve o menor percentual de redução $(6,04 \%)$ seguida do tratamento T4 $(7,81 \%)$. Independente do revestimento, associado aos antioxidantes e cloreto de cálcio, houve a inibição do escurecimento enzimático, ficando as amostras na tonalidade do amarelo.

Rojas-Graü, Tapia e Martín-Belloso (2008) obtiveram valores de tonalidade cromática semelhantes aos obtidos neste trabalho, ao avaliar amostras de maçã revestidas com alginato e gelana adicionadas de $\mathrm{N}$-acetilcisteína.

$\mathrm{Na}$ Tabela 5 são apresentados os valores de $\mathrm{pH}$ encontrados em maçãs minimamente processadas armazenadas a $4 \pm 1^{\circ} \mathrm{C}$ em um período de 15 dias.

Tabela 4. Ângulo de Hue ( $\left.\mathrm{h}^{*}\right)$ de maçãs minimamente processadas tratadas com diferentes revestimentos e armazenadas a $4 \pm 1^{\circ} \mathrm{C}$ durante 15 dias.

\begin{tabular}{ccccc}
\hline \multirow{2}{*}{ DIAS } & \multicolumn{4}{c}{ TRATAMENTO } \\
\cline { 2 - 5 } & T1 & T2 & T3 & T4 \\
\hline 0 & $100,51 \pm 0,21^{\mathrm{aB}}$ & $102,65 \pm 0,11^{\mathrm{aA}}$ & $102,65 \pm 0,07^{\mathrm{aA}}$ & $102,64 \pm 0,27^{\mathrm{aA}}$ \\
1 & $95,26 \pm 0,34^{\mathrm{bC}}$ & $98,36 \pm 0,74^{\mathrm{bB}}$ & $103,01 \pm 0,27^{\mathrm{aA}}$ & $102,64 \pm 0,16^{\mathrm{aA}}$ \\
3 & $94,79 \pm 0,31^{\mathrm{bC}}$ & $96,87 \pm 0,41^{\mathrm{eB}}$ & $102,38 \pm 0,64^{\mathrm{aA}}$ & $101,65 \pm 0,24^{\mathrm{bA}}$ \\
5 & $90,77 \pm 0,46^{\mathrm{cC}}$ & $99,42 \pm 0,15^{\mathrm{bB}}$ & $98,81 \pm 0,71^{\mathrm{bB}}$ & $101,98 \pm 0,39^{\mathrm{abA}}$ \\
7 & $91,52 \pm 0,38^{\mathrm{cD}}$ & $98,15 \pm 0,21^{\mathrm{cdB}}$ & $97,22 \pm 0,14^{\mathrm{cdC}}$ & $100,21 \pm 0,20^{\mathrm{cA}}$ \\
9 & $91,53 \pm 0,35^{\mathrm{cC}}$ & $98,93 \pm 0,27^{\mathrm{bcAB}}$ & $98,11 \pm 0,23^{\mathrm{bcB}}$ & $99,35 \pm 0,42^{\mathrm{dA}}$ \\
12 & $88,15 \pm 0,18^{\mathrm{dD}}$ & $97,08 \pm 0,24^{\mathrm{deB}}$ & $98,02 \pm 0,33^{\mathrm{bcA}}$ & $94,81 \pm 0,21^{\mathrm{eC}}$ \\
15 & $85,33 \pm 0,56^{\mathrm{eC}}$ & $93,89 \pm 0,27^{\mathrm{fB}}$ & $96,44 \pm 0,39^{\mathrm{dA}}$ & $94,62 \pm 0,12^{\mathrm{eB}}$ \\
\hline
\end{tabular}

Médias seguidas de mesma letra minúscula na coluna e maiúscula na linha não diferem entre si, pelo Teste de Tukey (P<0,05). (T1) controle; (T2) alginato $2 \%(\mathrm{~m} / \mathrm{v})$, ácido ascórbico $1 \%(\mathrm{~m} / \mathrm{v})$, ácido cítrico $0,25 \%$ (m/v), $\mathrm{CaCl}_{2} 1 \%(\mathrm{~m} / \mathrm{v})$, glicerol $1 \%$ (v/v); (T3) goma xantana $0,5 \%(\mathrm{~m} / \mathrm{v})$, ácido ascórbico $1 \%(\mathrm{~m} / \mathrm{v})$, ácido cítrico $0,25 \%(\mathrm{~m} / \mathrm{v}), \mathrm{CaCl}_{2} 1 \%$ (m/v), glicerol $1 \%(\mathrm{v} / \mathrm{v})$; (T4) goma tara $0,5 \%(\mathrm{~m} / \mathrm{v})$, ácido ascórbico $1 \%(\mathrm{~m} / \mathrm{v})$, ácido cítrico $0,25 \%(\mathrm{~m} / \mathrm{v}), \mathrm{CaCl}_{2} 1 \%(\mathrm{~m} / \mathrm{v})$, glicerol $1 \%(\mathrm{v} / \mathrm{v})$.

Fonte: Elaboração dos autores.

Tabela 5. $\mathrm{pH}$ de maçãs minimamente processadas tratadas com diferentes revestimentos e armazenadas a $4 \pm 1^{\circ} \mathrm{C}$ durante 15 dias.

\begin{tabular}{ccccc}
\hline DIAS & \multicolumn{5}{c}{ TRATAMENTOS } & \multicolumn{1}{c}{ T4 } \\
\hline 0 & $4,98 \pm 0,03^{\mathrm{aA}}$ & $4,79 \pm 0,05^{\mathrm{aB}}$ & $4,77 \pm 0,05^{\mathrm{aB}}$ & $4,77 \pm 0,03^{\mathrm{aB}}$ \\
1 & $4,97 \pm 0,05^{\mathrm{aA}}$ & $4,79 \pm 0,02^{\mathrm{aAB}}$ & $4,76 \pm 0,03^{\mathrm{aAB}}$ & $4,76 \pm 0,02^{\mathrm{abB}}$ \\
3 & $4,73 \pm 0,06^{\mathrm{bcA}}$ & $4,72 \pm 0,02^{\mathrm{aA}}$ & $4,72 \pm 0,03^{\mathrm{aA}}$ & $4,69 \pm 0,03^{\mathrm{abA}}$ \\
5 & $4,66 \pm 0,03^{\mathrm{cAB}}$ & $4,57 \pm 0,03^{\mathrm{bC}}$ & $4,72 \pm 0,02^{\mathrm{aA}}$ & $4,61 \pm 0,04^{\mathrm{abcBC}}$ \\
7 & $4,55 \pm 0,05^{\mathrm{dAB}}$ & $4,46 \pm 0,04^{\mathrm{cB}}$ & $4,49 \pm 0,03^{\mathrm{bB}}$ & $4,59 \pm 0,01^{\mathrm{abcA}}$ \\
9 & $4,70 \pm 0,02^{\mathrm{bcA}}$ & $4,40 \pm 0,01^{\mathrm{cdC}}$ & $4,47 \pm 0,05^{\mathrm{bBC}}$ & $4,53 \pm 0,02^{\mathrm{bcB}}$ \\
12 & $4,73 \pm 0,03^{\mathrm{bA}}$ & $4,34 \pm 0,03^{\mathrm{dC}}$ & $4,33 \pm 0,02^{\mathrm{cC}}$ & $4,42 \pm 0,03^{\mathrm{cdB}}$ \\
15 & $4,79 \pm 0,03^{\mathrm{bA}}$ & $4,33 \pm 0,01^{\mathrm{dB}}$ & $4,29 \pm 0,05^{\mathrm{cB}}$ & $4,26 \pm 0,04^{\mathrm{dB}}$ \\
\hline
\end{tabular}

Médias seguidas de mesma letra minúscula na coluna e maiúscula na linha não diferem entre si, pelo Teste de Tukey (P<0,05). (T1) controle; (T2) alginato $2 \%(\mathrm{~m} / \mathrm{v})$, ácido ascórbico $1 \%(\mathrm{~m} / \mathrm{v})$, ácido cítrico $0,25 \%$ (m/v), $\mathrm{CaCl}_{2} 1 \%$ (m/v), glicerol $1 \%$ (v/v); (T3) goma xantana $0,5 \%(\mathrm{~m} / \mathrm{v})$, ácido ascórbico $1 \%(\mathrm{~m} / \mathrm{v})$, ácido cítrico $0,25 \%(\mathrm{~m} / \mathrm{v}), \mathrm{CaCl}_{2} 1 \%(\mathrm{~m} / \mathrm{v})$, glicerol $1 \%$ (v/v); (T4) goma tara $0,5 \%(\mathrm{~m} / \mathrm{v})$, ácido ascórbico $1 \%(\mathrm{~m} / \mathrm{v})$, ácido cítrico $0,25 \%(\mathrm{~m} / \mathrm{v}), \mathrm{CaCl}_{2} 1 \%(\mathrm{~m} / \mathrm{v})$, glicerol $1 \%(\mathrm{v} / \mathrm{v})$.

Fonte: Elaboração dos autores. 
Houve tendência de redução do $\mathrm{pH}$ das amostras de maçãs minimamente processadas submetidas aos diferentes tratamentos. Ao término do armazenamento, o $\mathrm{pH}$ das amostras submetidas ao tratamento controle (T1) foi significativamente superior aos demais, sendo que este tratamento apresentou a menor redução do $\mathrm{pH}(3,8 \%)$. Este comportamento deve-se ao fato dos tratamentos T2, T3 e T4 serem adicionados de ácido cítrico e ascórbico. Dentre as amostras revestidas o tratamento T2 obteve a menor redução $(9,6 \%)$ e o T4 a maior $(10,7 \%)$.

Comportamento diferenciado foi observado por Fontes et al. (2008) em que as amostras de maçã revestidas com solução conservadora (ácidos cítrico e ascórbico, cloretos de sódio e cálcio), além de polímeros fécula de mandioca e àquelas com alginato, mantiveram o $\mathrm{pH}$ durante treze dias de armazenamento. Já o tratamento controle e naquelas revestidas com dextrina observou-se tendência ao aumento.

A acidez constitui fator de grande importância para o sabor e aroma dos frutos e, além disso, o $\mathrm{pH}$ influencia no escurecimento oxidativo dos tecidos vegetais. A diminuição do seu valor acarreta redução da velocidade de escurecimento do fruto (FREITAS, 2010).

$\mathrm{Na}$ Tabela 6 são apresentados os valores de sólidos solúveis totais (Brix) encontrados em maçãs minimamente processadas armazenadas em $4 \pm 1{ }^{\circ} \mathrm{C}$ em um período de 15 dias.

Tabela 6. Sólidos solúveis totais $\left({ }^{\circ}\right.$ Brix) encontrados em maçãs minimamente processadas tratadas com diferentes revestimentos e armazenadas a $4 \pm 1^{\circ} \mathrm{C}$ durante 15 dias.

\begin{tabular}{ccccc}
\hline DIAS & T1 & T2 $^{\text {TRATAMENTO }}$ & T3 \\
\hline 0 & $11,80 \pm 0,1^{\mathrm{gA}}$ & $11,60 \pm 0,1^{\mathrm{eB}}$ & $11,60 \pm 0,1^{\mathrm{eB}}$ & $11,60 \pm 0,1^{\mathrm{fB}}$ \\
1 & $11,90 \pm 0,1^{\mathrm{fA}}$ & $11,60 \pm 0,1^{\mathrm{eC}}$ & $11,70 \pm 0,2^{\mathrm{eB}}$ & $11,70 \pm 0,1^{\mathrm{eB}}$ \\
3 & $13,90 \pm 0,2^{\mathrm{dA}}$ & $12,00 \pm 0,2^{\mathrm{dC}}$ & $13,30 \pm 0,1^{\mathrm{cB}}$ & $13,30 \pm 0,2^{\mathrm{bB}}$ \\
5 & $14,30 \pm 0,1^{\mathrm{cA}}$ & $12,30 \pm 0,1^{\mathrm{cB}}$ & $14,00 \pm 0,1^{\mathrm{abA}}$ & $13,90 \pm 0,1^{\mathrm{aA}}$ \\
7 & $14,90 \pm 0,1^{\mathrm{bA}}$ & $12,60 \pm 0,1^{\mathrm{bD}}$ & $14,50 \pm 0,2^{\mathrm{aB}}$ & $13,90 \pm 0,1^{\mathrm{aC}}$ \\
9 & $13,90 \pm 0,2^{\mathrm{dA}}$ & $10,60 \pm 0,2^{\mathrm{fC}}$ & $13,90 \pm 0,1^{\mathrm{bA}}$ & $12,60 \pm 0,1^{\mathrm{cB}}$ \\
12 & $13,30 \pm 0,1^{\mathrm{eA}}$ & $12,00 \pm 0,1^{\mathrm{dB}}$ & $13,30 \pm 0,1^{\mathrm{cA}}$ & $12,00 \pm 0,2^{\mathrm{dB}}$ \\
15 & $15,30 \pm 0,2^{\mathrm{aA}}$ & $13,60 \pm 0,2^{\mathrm{aB}}$ & $12,60 \pm 0,2^{\mathrm{dC}}$ & $12,00 \pm 0,1^{\mathrm{dD}}$ \\
\hline
\end{tabular}

Médias seguidas de mesma letra minúscula na coluna e maiúscula na linha não diferem entre si, pelo Teste de Tukey $(\mathrm{P}<0,05)$. (T1) controle; (T2) alginato $2 \%(\mathrm{~m} / \mathrm{v})$, ácido ascórbico $1 \%(\mathrm{~m} / \mathrm{v})$, ácido cítrico $0,25 \%(\mathrm{~m} / \mathrm{v}), \mathrm{CaCl}_{2} 1 \%(\mathrm{~m} / \mathrm{v})$, glicerol $1 \%$ (v/v); (T3) goma xantana $0,5 \%$ (m/v), ácido ascórbico $1 \%(\mathrm{~m} / \mathrm{v})$, ácido cítrico 0,25\% (m/v), $\mathrm{CaCl}_{2} 1 \%$ (m/v), glicerol $1 \%$ (v/v); (T4) goma tara $0,5 \%(\mathrm{~m} / \mathrm{v})$, ácido ascórbico $1 \%(\mathrm{~m} / \mathrm{v})$, ácido cítrico 0,25\% (m/v), $\mathrm{CaCl}_{2} 1 \%(\mathrm{~m} / \mathrm{v})$, glicerol $1 \%(\mathrm{v} / \mathrm{v})$.

Fonte: Elaboração dos autores.

Os valores de sólidos solúveis totais oscilaram durante o armazenamento, com tendência de aumento. A amostra controle foi a que obteve o maior aumento $(22,8 \%)$ e a amostra revestida com goma tara o menor $(3,3 \%)$, durante os 15 dias de armazenamento. Estes resultados mostram que os tratamentos com as gomas impediram de certa forma o amadurecimento da fruta, principalmente o revestimento com goma tara. $\mathrm{O}$ aumento observado nos sólidos solúveis totais até o $7^{\circ}$ dia e após o $12^{\circ}$ pode estar relacionado ao acúmulo de açúcares pela perda da umidade (COSTA; BALBINO, 2002), já nos dias 9 e 12 houve redução, este comportamento pode estar associado ao consumo de açúcares, devido ao maior metabolismo respiratório da fruta, e ao crescimento de micro-organismos. Entretanto tais fenômenos ocorrem, simultaneamente, com diferenças de intensidade dependendo do período. 
Não foi detectada a presença de coliformes totais e Escherichia coli $\left(<10^{2} \mathrm{UFC}^{-g^{-1}}\right)$, assim como de Salmonella (ausência em 25g) nas amostras de maçãs minimamente processadas, confirmando a eficiência dos cuidados higiênicos e da ação do cloro orgânico na desinfecção das amostras.

No Brasil, ainda não existe legislação específica para frutas e hortaliças minimamente processadas com os limites de contagens tolerados. Entretanto, existe legislação para frutas frescas, in natura, preparadas (descascadas ou selecionadas ou fracionadas) sanitizadas, refrigeradas ou congeladas, que estipula valores máximos de coliformes termotolerantes de $5 \times 10^{2}$ UFC.g $^{-1}$ e ausência de Salmonella em $25 \mathrm{~g}$ de amostra (BRASIL, 2001).
A Figura 1 mostra as curvas de crescimento para os micro-organismos psicrófilos encontrados em maçãs minimamente processadas submetidas aos diferentes tratamentos com os revestimentos comestíveis e armazenadas à $4 \pm 1{ }^{\circ} \mathrm{C}$ por 15 dias.

$\mathrm{Na}$ análise de psicrófilos, o tratamento controle foi o que obteve a maior contagem inicial $(2,27$ $\log$ UFC. $\mathrm{g}^{-1}$ ) quando comparado aos diferentes tratamentos com revestimento. O T4 ao longo dos dias de armazenamento foi o que obteve maior desenvolvimento log até o décimo segundo dia (Figura 1). Os tratamentos T2 e T3 foram os que obtiveram menor crescimento microbiano em 15 dias de análise.

Figura 1. Curvas de crescimento microbiano para os micro-organismos psicrófilos encontrados em maçãs minimamente processadas armazenadas à $4 \pm 1^{\circ} \mathrm{C}$ por 15 dias utilizando diferentes revestimentos comestíveis.

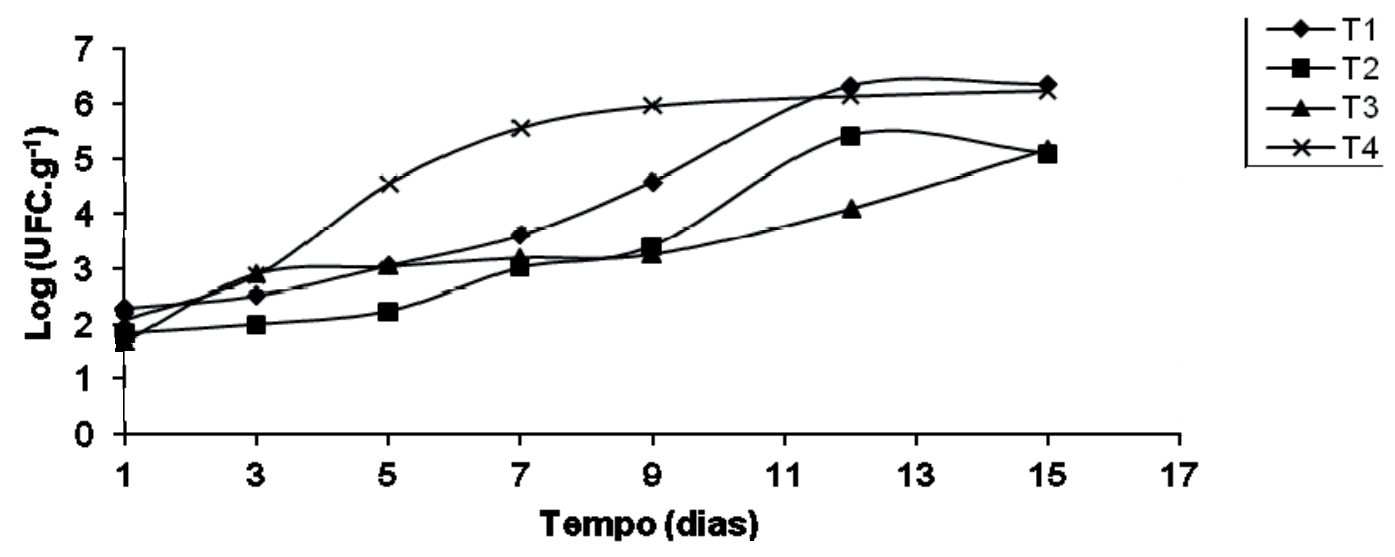

Fonte: Elaboração dos autores.

Olivas, Mattinson e Barbosa-Cánovas (2007) avaliaram maçãs minimamente processadas revestidas com alginato de sódio estocadas a 4 ${ }^{\circ} \mathrm{C}$. De acordo com seus resultados, foram obtidos baixos valores para psicrófilos $\left(1 \times 10^{1} \mathrm{UFC}_{\mathrm{g}} \mathrm{g}^{-1}\right) \mathrm{e}$ bolores e leveduras $\left(<10^{1} \log \mathrm{UFC} . \mathrm{g}^{-1}\right)$ em 15 dias de armazenamento, valores estes inferiores aos obtidos neste trabalho, tanto para psicrófilos, $\left(10^{5} \mathrm{UFC} \cdot \mathrm{g}^{-1}\right)$ (Figura 1) como para bolores e leveduras $\left(10^{5}\right.$ $\mathrm{UFC}^{-1} \mathrm{~g}^{-1}$ ) (Figura 2) em 12 dias de armazenamento quando tratadas com alginato de sódio. 
Figura 2. Curvas de crescimento microbiano para bolores e leveduras encontrados em maçãs minimamente processadas armazenadas em $4 \pm 1{ }^{\circ} \mathrm{C}$ por 15 dias utilizando diferentes revestimentos comestíveis.

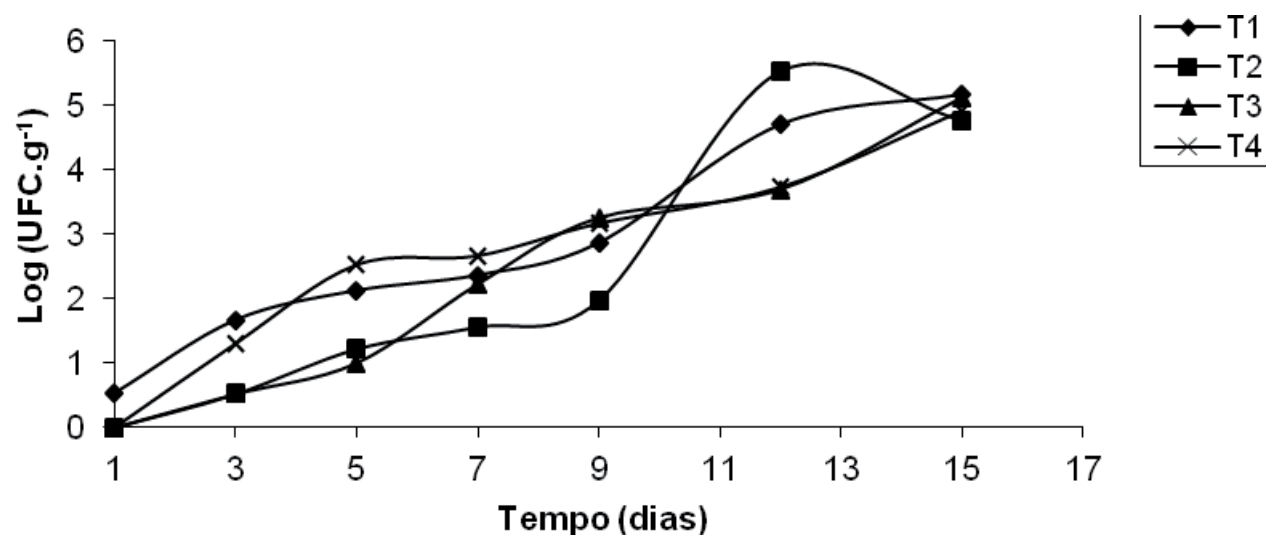

Fonte: Elaboração dos autores.

Rojas-Graü, Tapia e Martín-Belloso (2008) mostraram que as amostras de maçãs revestidas com alginato e gelana apresentaram menor crescimento de psicrófilos que a amostra controle, alcançando $10^{5}$ UFC. $\mathrm{g}^{-1}$, em 21 dias de armazenamento refrigerado, resultados semelhantes aos obtidos neste estudo.

A Figura 2 mostra as curvas de crescimento microbiano para bolores e leveduras encontrados em maçãs minimamente processadas armazenadas à $4 \pm 1{ }^{\circ} \mathrm{C}$ por 15 dias.

Pode-se observar que todos os tratamentos nos quais foram utilizadas as diferentes gomas, a contagem inicial foi menor do que a contagem encontrada para a amostra controle (0,52 Log UFC. $\left.\mathrm{g}^{-1}\right)$. Todos os tratamentos apresentaram comportamento semelhante, isto é com o passar dos dias de armazenamento houve aumento da contagem de bolores e leveduras. $\mathrm{O}$ tratamento $\mathrm{T} 3$ e T4 foram os que obtiveram o menor crescimento em 12 e 15 dias de armazenamento em relação aos outros tratamentos.

Freitas (2010) avaliaram maçãs minimamente processadas demonstrando que a adição de goma xantana estimulou o crescimento de bolores e leveduras quando comparado ao controle. Estes resultados indicam que o revestimento tornou-se um bom substrato para o desenvolvimento de microorganismos. Entretanto, quando utilizada a goma xantana associado à solução conservadora (ácido ascórbico e cítrico) houve redução do crescimento destes micro-organismos, assim como observado neste trabalho.

\section{Conclusão}

Pode-se concluir que os diferentes revestimentos utilizados neste trabalho foram eficientes na conservação de maçãs minimamente processadas, quando comparado a amostra controle. Dos revestimentos avaliados, o tratamento com goma xantana, associado aos ácidos cítrico, ascórbico e cloreto de cálcio foi o que apresentou os melhores resultados, mostrando-se capaz de reduzir a perda de massa, o crescimento microbiano, manter a cor e a firmeza.

\section{Referências}

ASSOCIATION OF OFFICIAL ANALYTICAL CHEMISTS - AOAC. Official methods of analysis. $16^{\text {th }}$ ed. Washington, D.C, 2000.

AMERICAN PUBLIC HEALTH ASSOCIATION APHA. Compendium of methods for the microbiological examination of foods. Washington, D.C, 2001. 676 p. 
BRASIL. Ministério da Saúde. Resolução RDC nº 12, de 02 de janeiro de 2001. Aprova o Regulamento Técnico sobre padrões microbiológicos para alimentos. Diário Oficial [da] República Federativa do Brasil, Brasília, DF, 10 jan. 2001. Seção 1, p. 46-53, 2001.

CARDOSO, W. S.; PINHEIRO, F. A.; PATELLI, T.; PEREZ, R.; RAMOS, A. M. Determinação da concentração de sulfito para a manutenção da qualidade da cor em maçã desidratada. Revista Analytica, São Paulo, n. 29, p. 127-132, 2007.

CARVALHO, A. V.; LIMA, L. C. O. Qualidade de kiwis minimamente processados e submetidos a tratamento com ácido ascórbico, ácido cítrico e cloreto de cálcio. Pesquisa Agropecuária Brasileira, Brasília, v. 37, n. 5, p. 679-685, 2002.

COSTA, A. F. S.; BALBINO, J. M. S. Características da fruta para exportação e normas de qualidade. In: FOLEGATTI, M. I. S.; MATSUURA, F. C. A. U. (Ed.). Mamão: pós-colheita. Brasília, DF: Embrapa Informação Tecnológica, 2002. p. 12-18. (Série Frutas do Brasil, 21).

FONTES, L. C. B. Uso de solução conservadora e de películas comestivieis em maçãs da cultivar Royal Gala minimamente processadas: efeito na fisiologia e na conservação. 2005. (Dissertação em Ciência dos Alimentos), Universidade de São Paulo, São Paulo.

FONTES, L. C. B.; SARMENTO, S. B. S. S.; SPOTO, M. H. F.; DIAS, C. T. S. Conservação de maçã minimamente processada com o uso de películas comestíveis. Ciência e Tecnologia de Alimentos, Campinas, v. 28, n. 28, p. 872880, 2008.

FREITAS, I. R. Goma xantana como carreadora de solução conservadora e cloreto de cálcio aplicado a maçã minimamente processada. 2010. Monografia (Especialização em Ciência dos Alimentos) Universidade Federal de Pelotas, Pelotas.

FOOD STANDARDS AUSTRALIA NEW ZEALAND - FSANZ. Tara gum as a food additive. Food Standars. New Zealand, Austrália, 2006.

GARCIA, E.; BARRET, D. M. Preservative treatments for fresh cut fruits and vegetables. In: LAMIKANRA, O. (Ed.). Fresh-cut fruits and vegetables. Florida: CRC Press, 2002. p. 267-304.

GARCÍA-OCHOA, F.; SANTOS, V. E.; CASAS, J. A.; GÓMEZ, E. Xanthan gum: production, recovery and properties. Biotechnology Advances, United States, v. 18, n. 7, p. 549-579, 2000.

JUNQUEIRA, M. S.; SOARES, N. F. F.; REIS, R. C.; CARNEIRO, J. D. S.; BENICIOS, R. T.; YOKOTA,
S. R. C. Efeito de embalagens ativas no escurecimento enzimático de batatas (Solanum tuberosum) fatiadas e minimamente processadas. Semina: Ciências Agrárias, Londrina, v. 30, n. 3, p. 613-618, 2009.

KROCHTA, J. M.; MULDER-JOHNSTON, C. Edible and biodegradable polymer films:challenges and opportunities. Food Technology, Chicago, v. 51, n. 2, p. 61-74, 1997.

MENEGUEL, R. F. A.; BENASSI, M. T.; YAMASHITA, F. Revestimento comestível de alginato de sódio para frutos de amora-preta (Rubus ulmifolius). Semina: Ciências Agrárias, Londrina, v. 29, n. 3, p. 609-618, 2008.

OLIVAS, G. I.; MATTINSON, D. S.; BARBOSACÁNOVAS, G. V. Alginate coatings for preservation of minimally processed 'Gala' apples. Postharvest Biology and Technology, Amsterdam, v. 45, n. 1, p. 89-96, 2007.

OUATTARA, B.; SABATO, S. F.; LACROIX, M. Combined effect of antimicrobial coating and gamma irradiation on shelf life extension of pre-cooked shrimp (Penaeus spp.). International Journal of Food Microbiolology, Netherlands, v. 68, n. 2, p. 1-9, 2001.

PERERA，N.; GAMAGE，T. V.; WAKELING，L.; GAMLATH, G. G. S.; VERSTEEG, C. Colour and texture of apples high pressure processed in pineapple juice. Innovative Food Science and Emerging Technologies, Berlin, v. 11, n. 3, p. 39-46, 2010.

PEREZ-GAGO, M. B.; SERRA, M.; DEL-RÍO, M. Color changes of fresh-cut apples coated with why protein concentrate-based edible coatings. Postharvest Biology and Technology, Amsterdam, v. 39, n. 1, p. 84-92, 2006.

QI, H.; HU, W.; JIANG, A.; TIAN, M. Extending shelflife of fresh-cut 'Fuji' apples with chitosan-coatings. Innovative Food Science \& Emerging Technologies, Berlin, v. 12, n. 1, p. 62-66, 2011.

ROJAS-GRAÜ, M. A.; TAPIA, M. S.; MARTÍNBELLOSO, O. Using polysaccharide-based edible coatings to maintain quality of fresh-cut Fuji apples. LWT - Food Science and Techonology, London, v. 41, n. 1, p. 139-147, 2008.

ROJAS-GRAÜ, M. A.; RAYBAUDI-MASSILIA, R. M.; SOLIVA-FORTUNY, R. C.; AVENA-BUSTILLOS, R. J.; MCHUGH, T. H.; MARTÍN-BELLOSO, O. Apple puree-alginaté edible coating as carrier of antimicrobial agents to prolong shelf-life of fresh-cut apples. Postharvest Biology and Technology, Amsterdam, v. 45, n. 3, p. 254-264, 2007. 
SITTIKIJYOTHIN, W.; SAMPAIO, P.; GONÇALVES, M. P. Heat-induced gelation of b-lactoglobulin at varying $\mathrm{pH}$ : Effect of tara gum on the rheological and structural properties of the gels. Food Hydrocolloids, Oxford, v. 21, n. 3, p. 1046-1055, 2007.

SITTIKIJYOTHIN, W.; TORRES, D.; GONÇALVES, M. P. Modelling the rheological behaviour of galactomannan aqueous solutions. Carbohydrate Polymers, Barking, v. 59 , n. 3, p. 339-350, 2005.
SOUZA, B. S.; DURIGAN, J. F.; DONADON, J. R.; LIMA, M. A. Qualidade e comportamento do mamão 'formosa' minimamente processado. Brazilian Journal of Food Technology, Campinas, v. 8, n. 3, p. 243-247, 2005.

VILAS BOAS, E. V. B.; REIS, C. M. F.; MELO, A. A.; MARTINS, B. Uso de misturas químicas para manutenção da firmeza de banana 'prata' minimamente processada. Ciência e agrotecnologia, Lavras, v. 1, n. 33, p. 237-244, 2009.

XUEWU, Z.; XIN, L.; DEXIANG, G.; WEI, Z.; TONG, X.; YONGHONG, M. Rheological models for xanthan gum. Journal of Food Engineering, Essex, v. 27, n. 2, p. 203-209, 1996. 